\title{
Questes
}

Revue pluridisciplinaire d'études médiévales

42 | 2021

Fama : réputation et renommée

\section{Réflexions sur la debonnaireté dans l'editio princeps de Lancelot du Lac}

\section{Karine Dicharry}

\section{(2) OpenEdition}

1 Journals

\section{Édition électronique}

URL : https://journals.openedition.org/questes/5692

DOI : $10.4000 /$ questes.5692

ISSN : 2109-9472

Éditeur

Les Amis de Questes

\section{Édition imprimée}

Date de publication : 28 janvier 2021

Pagination : $67-79$

ISSN : 2102-7188

\section{Référence électronique}

Karine Dicharry, «Réflexions sur la debonnaireté dans l'editio princeps de Lancelot du Lac », Questes

[En ligne], 42 | 2021, mis en ligne le 28 février 2021, consulté le 06 février 2022. URL : http:// journals.openedition.org/questes/5692 ; DOI : https://doi.org/10.4000/questes.5692

\section{(C) Association des amis de «Questes »}




\title{
Réflexions sur la debonnaireté dans l'editio princeps de Lancelot du Lac
}

\author{
Karine DICHARRY \\ Université de Lille - Laboratoire Alithila
}

Leur omniprésence dans les textes antiques en témoigne : la réputation et la renommée sont considérées comme des valeurs essentielles depuis l'Antiquité, car, selon Jean-Pierre Neraudau, la fama « garantit, quand elle est bonne, l'accès à l'estime des hommes, puis à leur mémoire ${ }^{1} »$. Ces principes sont également revendiqués, à l'aube de la modernité, dans le prologue de l'editio princeps de Lancelot $d u L a c^{2}$, où l'éditeur affirme l'importance de célébrer les hauts faits des hommes valeureux et la vertu pédagogique des récits qui en font l'éloge :

Il est bien decent et raisonnable que [...] les vertus et glorieux faiz des excellentz hommes vivent aprez mort et soient aux successeurs en perpetuelle memoire, et que a chascun soit fait memorial condig[n]e selon ses valeur, merite, et estat, aux ungz par sculptures et descriptions sur leur monuementz, et aux autres par rediger en

\footnotetext{
${ }^{1}$ Jean-Pierre Néraudau, «La Fama dans la Rome antique », Médiévales, n 24, 1993, «La renommée », p. 27-34, cit. p. 30.

${ }^{2}$ Il s'agit du tout premier roman arthurien imprimé. Fruit de l'association de deux éditeursimprimeurs, Jean Le Bourgeois (Rouen) et Jean Du Pré (Paris), et produit à la fin de l'année 1488, il présente en deux volumes la fin du Lancelot-Graal, cycle romanesque médiéval le plus illustre, à savoir le Lancelot, La Queste del saint Graal et La Mort Artu et inaugure la « vogue éditoriale de la littérature chevaleresque médiévale à la Renaissance » (Gabrielle Burg, «De Paris à Lyon, les mutations éditoriales du Lancelot du Lac», Carte Romanze, 3/1, 2015, p. 287) en servant de base aux neuf éditions ultérieures qui s'échelonnent jusqu'à 1591. La recherche actuelle tend à réhabiliter les versions tardives des cuvres du Moyen Âge, notamment parce que «c'est essentiellement le passage à l'imprimé qui assure la postérité littéraire de ces proses » et parce que «c'est à partir de la forme imprimée de ces romans en prose au tournant entre Moyen Âge et Renaissance que se forgera l'imaginaire médiéval de la littérature moderne » (Le Roman français dans les premiers imprimés, dir. Anne Schoysman et Maria Colombo Timelli, Paris, Classiques Garnier, 2016, introduction des éditrices, p. 10 et 8).
} 
volumes leurs excellences, triumphes, et operations, qui sont et demeurent a toujours en bon exemple aux lecteurs et aux auditeurs, car ce qui y est recité de bien et de vertu est ensaignement et excitation de bien et vertueusement vivre pour en acquerir renommée perpetuelle entre les hommes, oultre l'esperee remuneration eternelle ${ }^{3}$.

Mais sur quoi se fondent et se construisent la réputation et la renommée dans l'incunable? Quelles sont les valeurs exaltées ? Se résument-elles à la prouesse chevaleresque, aptitude physique, exercice de la violence institutionnalisée, ou participent-elles d'un système de valeurs, d'une véritable éthique dont Lancelot constituerait le modèle? Surtout, quels rapports entretiennent-elles avec les valeurs chevaleresques, $1^{1}$ aristocratie $^{4}$ et le concept de la debonnaireté ?

\section{Qu'est-ce que la debonnaireté ?}

Notion spécifiquement médiévale, aujourd'hui disparue, elle signifie d'abord « de bonne origine, de bonne race, noble ${ }^{5} »$. Par extension, elle désigne les qualités de celui qui est noble : des qualités morales telles que la douceur, la générosité, l'amabilité, la gentillesse, l'affabilité et la bonté, et des qualités guerrières de vaillance et de courage.

\footnotetext{
${ }^{3}$ Lancelot du Lac (1488), prologue, vol. 1, pdf p. 9. Toutes les références au princeps renvoient aux fichiers pdf des exemplaires de la BNF cotés RES-Y2-46 (vol. 1) et RES-Y2-47 (vol. 2), disponibles en ligne sur le site Gallica aux adresses suivantes : http://gallica.bnf.fr/ark:/12148/bpt6k111059t?rk=21459;2 http://gallica.bnf.fr/ark:/12148/bpt6k111060r?rk=42918;4 (pages consultées le 18 juin 2018). Nous soulignons. Le texte est encore inédit et nous en préparons l'édition. Comme ces exemplaires présentent des feuillets déplacés, nous indiquerons systématiquement les pages de la numérisation, afin de permettre au lecteur de s'y reporter plus aisément.

${ }^{4}$ Sur les rapports entre noblesse et chevalerie, voir par exemple Jean Flori, «Aristocratie et valeurs chevaleresques dans la seconde moitié du XII ${ }^{\mathrm{e}}$ siècle : l'exemple des lais de Marie de France », Le Moyen Âge, vol. 96, 1990, p. 35-65.

${ }^{5}$ Dans son Dictionnaire de l'ancienne langue française et de tous ses dialectes $d u$ IX au $X V^{e}$ siècle (1881), Frédéric Godefroy indique que le substantif aire a, "par extension, signifié la race, l'extraction, et par suite toutes les qualités, les dispositions, bonnes ou mauvaises ».
} 
On ne trouve dans l'editio princeps de Lancelot du Lac que trois des termes relevant du paradigme morphologique de debonnaire ${ }^{6}$ : l'adjectif épicène debonnaire, le substantif debonnaireté et l'adverbe debonnairement, avec, toutes formes confondues, 105 occurrences $^{7}$. Le relevé de la fréquence d'occurrences des termes du paradigme sémantique de debonnaire confirme de manière quantitative les impressions qui se dégagent de la lecture de l'œuvre ${ }^{8}$. Ce sondage fait ainsi apparaître l'importance de la présence du lexique des valeurs aristocratiques, principalement dans le domaine moral, parmi lesquelles figurent en bonne place debonnaire et ses dérivés. Ce vocabulaire est lié au thème de l'œuvre, aussi l'on enregistre un plus grand nombre d'emplois dans le premier volume où ce thème est prépondérant. En effet, le premier comporte les deux premières parties du Lancelot en prose, de la naissance du personnage éponyme jusqu'à son départ pour Corbenic. Il présente donc les aventures chevaleresques du héros et des chevaliers de la Table Ronde. Le deuxième volume rapporte la fin du Lancelot ainsi que la Queste del Saint Graal et La Mort le roi Artu. Par

${ }^{6}$ Pour une idée plus précise de l'étendue de ce paradigme, on consultera, notamment, le Dictionnaire $d u$ Moyen Français accessible en ligne : http://www.cnrtl.fr/definition/dmf/d\%C3\%A9bonnairet\%C3\%A9?idf=xxx;etym=ager ;

Olivier Bertrand et Silvère Menegaldo, Vocabulaire d'ancien français. Fiches à l'usage des concours, Paris, Armand Colin, 2006 ; Roland Guillot, L'Épreuve d'ancien français aux concours. Fiches de vocabulaire, Paris, Champion, coll. «Unichamp-Essentiel», 2008 ; Laurence Hélix, Parcours méthodique. L'épreuve de vocabulaire d'ancien français. Fiches de sémantique, Paris, Éditions du Temps, 1999.

${ }^{7}$ Dans le vol. I, 21 occurrences de l'adjectif, 29 du substantif et 11 de l'adverbe, soit $58 \%$ des emplois. Dans le vol. II, 22 occurrences de l'adjectif, 13 du substantif et 9 de l'adverbe, soit $42 \%$. Les pourcentages sont arrondis au demi-point le plus proche.

${ }^{8}$ Ainsi, noble, noblesse, noblement apparaissent à 51 reprises (36/15), preu 107 fois (99/8), large, largesse, largement 56 (35/21), franc, franchise, franchement 41 (17/24), courtois, courtoisement 66 (44/22), vaillant, vaillamment, 133 (87/46), doux, doulcement 135 (77/58) et gent, gentil 89 (67/22). Notre prélèvement a volontairement exclu preudhomme, quelle qu'en soit la graphie (ainsi que le féminin preude femme), dont la surreprésentation dans les œuvres médiévales d'inspiration courtoise aurait obscurci les résultats. De même, nous avons écarté les paradigmes morphologiques de bel (belle, beau, bellement...) et de hault (haulte, haultement ...), dont l'importante polysémie entraîne une multiplication des emplois et aboutit à une récurrence peu représentative. Nous indiquerons systématiquement le nombre total d'occurrences et entre parenthèses le détail pour le vol. I puis le vol. II. Nous ne signalons pas toutes les variations graphiques, mais elles ont été prises en compte, comme les flexions de genre et de nombre. 
conséquent, les prouesses chevaleresques y tiennent une moindre place, en particulier dans la Queste, où les valeurs spirituelles de la chevalerie célestielle supplantent celles, plus courtoises, de la chevalerie terrienne; de même dans la dernière partie, où, minée par les jalousies et les haines, l'institution de la Table Ronde se délite jusqu'à la destruction totale du royaume d'Arthur et de ses grandes figures ${ }^{9}$.

Il convient à présent de déterminer le périmètre sémantique de l'adjectif debonnaire et de ses dérivés dans l'œuvre afin d'affiner notre analyse. Leur présence dans des énumérations fournit une contextualisation qui permet de mieux cerner la signification du terme. La debonnaireté apparaît comme une des trois qualités fondamentales de la chevalerie : «vous avés failly a troys des meilleures vertus qui puissent estre en chevalier, car vous n'avez ne sens, ne debonnaireté, ne courtoisie. ${ }^{10} »$. Dans ces propos adressés au roi Claudas, il apparaît que les qualités énoncées sont attendues d'un roi aussi bien que d'un chevalier, c'est-à-dire qu'elles concernent l'ensemble de l'aristocratie. Les autres vertus du triptyque, peut-être inspiré des trois vertus théologales ${ }^{11}$, connaissent une légère variation dans l'œuvre, «trois vertus souffisantes a toutes choses terriennes mettre au dessoubz : c'est debonnaireté, largesse et magnanimité. ${ }^{12}{ }^{\prime}$, dans le discours tenu par Claudas, dont la debonnaireté est, nous le verrons plus loin, sujette à caution. Ainsi, cette qualité est unanimement reconnue à défaut d'être uniment pratiquée. La notion apparaît également dans des énumérations plus étendues de vertus :

\footnotetext{
${ }^{9}$ C'est particulièrement flagrant dans le relevé du paradigme morphologique preu, par exemple, dont on dénombre 99 occurrences dans le volume I et seulement 8 dans le volume II.

${ }^{10}$ Vol. I, col. 75a, p. 41.

${ }^{11}$ Pour mémoire, il s'agit de la foi, de l'espérance et de la charité. Dans ses Étymologies, Isidore de Séville fait dériver vir de virtus ; même s'il inverse le rapport logique entre les deux termes, il signale ainsi que la vertu est d'abord une qualité virile. Avec les quatre vertus cardinales que sont la prudence, la tempérance, la force d'âme et la justice (II, 24,5), elles constituent les vertus catholiques. Il recense également les deux vertus royales essentielles : la justice et la piété (IX, $3,5)$.

${ }^{12}$ Vol. I, col. 85a, p. 62.
} 
Il m'est advis que tel peut avoir les bontez du cueur qui ne peut mie avoir celles du corps, car tel est courtois, sage, debonnaire, loyal, piteux, large, et hardy - ce sont les vertus du cueur - qui toutesfois ne peut pas estre corsu, fort, legier, beau, ne plaisant. Toutes ces bontez sont du corps, et l'omme les apporte avec lui du ventre sa mere dés l'eure qu'il est né, mais les vertus du cueur pourroit-il avoir, se m'est adviz, se negligence ne l'empeschoit, car chacun peut avoir courtoisie et debonnaireté, et les autres biens qui du cueur descendent ${ }^{13}$.

Dans cet extrait du dialogue entre Lancelot et la Dame du Lac avant qu'il ne se rende à la cour d'Arthur pour être adoubé, le futur héros dresse l'inventaire des qualités morales (dont la debonnaireté) et physiques que doit posséder un chevalier. La fée complète la liste en rappelant une condition sine qua non de l'accès à la classe guerrière, le droit de election, l'appartenance à l'aristocratie :

Au commencement de l'ordre de chevalerie, il fut dit a celui qui vouloit chevalier estre et qui le don en avoit par droit de election qu'il fut courtois sans villennie, debonnaire sans follie, piteux vers les souffreteux, large et appareillié de secourier les indigens, prest et entalenté de destruire les robeurs et les meurdriers, de droit juger sans amour et sans haine ${ }^{14}$.

L'exposé sur l'histoire de la chevalerie souligne l'importance de la mesure, de la tempérance. En somme, le terme entre dans la liste des attributs du chevalier pour constituer une définition de l'éthique chevaleresque ou aristocratique : «Or regardez tous les biens que chevalier peut avoir : c'est force, hardiesse, beauté, gentillesse, debonnaireté, courtoisie, largesse, et force d'avoir et d'amis. ${ }^{15} \gg$. Cette propriété, dont la place ici ne semble pas obéir à une hiérarchie des valeurs, est visiblement une qualité morale qui connote la bonté, la noblesse, une certaine forme d'éducation, un comportement conforme aux valeurs du groupe social auquel il appartient et à la mission qui lui est assignée. Les doublets synonymiques

\footnotetext{
${ }^{13}$ Ibid., col. 144b-145a, p. 75-76. Nous soulignons.

${ }^{14}$ Ibid., col. 145a-146b, p. 76.

${ }^{15}$ Ibid., col. 966 b, p. 490.
} 
permettent de préciser davantage l'acception du terme. Ainsi, on relève des binômes avec large $(\text { sse })^{16}$, prouesse ${ }^{17}$, piteux (compatissant)/pitié ${ }^{18}$, gentil ${ }^{19}$, courtois $(\text { ie })^{20}$, doulx/doulce $(u r)^{21}$, et franchise (au sens de loyauté, droiture) ${ }^{22}$. Ces associations confirment non seulement la place de la debonnaireté dans le système de valeurs chevaleresques et aristocratiques, mais aussi l'étendue de son spectre polysémique. Par ailleurs, les termes de la famille de debonnaire apparaissent dans des constructions antithétiques qui aident à en percevoir les limites. Ainsi, la debonnaireté est opposée à la force : dans les couples antinomiques tels que «par force [/] par debonnaireté23 $»$ ou dans l'expression «par prouesse d'armes ou par debonnaireté ${ }^{4}$ ». On retrouve également ces phénomènes antithétiques dans des parallélismes de construction comme «ains a esté debonnaire et piteux, mais d'ores en avant seras-tu felon et cruel $^{25} »$, « elle fut fort felonne et luy devoit estre debonnaire ${ }^{26} »$ ou encore « a celui a qui je devroye estre plus debonnaire seray-je plus mauvaise ${ }^{27} \gg$. Finalement, le périmètre sémantique du paradigme

\footnotetext{
16 « sa largesse et sa debonnaireté », ibid., col. 51a, p. 29 et col. 52b, p. 29.

17 « les grandes prouesses de son seigneur et les grans debonnairetez », ibid., col. 31a, p. 19.

18 «tant est piteux et debonnaire », ibid., col. 69a, p. 38 ; « me cuides-tu a si debonnaire et a si piteux ? », ibid., col. 133a, p. 54 ; « ains as esté debonnaire et piteux », vol. II, col. 277a, p. 139 ; «tu es homme sans pitié et sans debonnaireté », ibid., col. 277a, p. 139.

${ }^{19}$ «le plus gentil roy et le plus debonnaire du monde», vol. I, col. 75a, p. 41 ; «moult estoit gentil et debonnaire », ibid., col. 518b, p. 262.

${ }^{20}$ « ne debonnaireté, ne courtoisie », ibid., col. 75a, p. 41 ; « ne debonnaire, ne courtois », ibid., col. 75a, p. 41 ; « courtois et debonnaire », ibid., col. 75a, p. 41 ; « courtoisie et debonnaireté », ibid., col. 145a, p. 76 ; « le plus debonnaire et le plus courtois », ibid., col. 806b, p. 406 ; «moult estoient les gens courtois et debonnaires », vol. II, col. 473a, p. 237.

${ }^{21}$ «a debonnaireté et a doulceur», vol. I, col. 147a, p. 77 ; «celle qui moult estoit doulce et debonnaire », ibid., col. 605a, p. 306 ; « doulx et debonnaire», ibid., col. 909a, p. 458 ; « doulce et debonnaire », vol. II, col. 477a, p. 239.

22 « ains avoit toute debonnaireté et toute franchise mise arriere », vol. I, col. 557a, p 282 ; « par sa franchise et par sa debonnaireté », ibid., col. 818b, p. 412.

${ }^{23}$ Ibid., col. 570b, p. 288 ; ou « faire debonnairement [/] faire force », Vol. II, col. 14b, p. 9, et la variation « debonnairement [/] face force », ibid., col. 237a, p. 119.

${ }^{24}$ Ibid., col. 327a, p. 164. La conjonction disjonctive, exprimant une alternative, oppose les deux éléments, la force physique et la bonté morale, même si la prouesse d'armes entre dans le cadre de la violence institutionnalisée, c'est-à-dire considérée comme légitime dans le contexte guerrier.
}

${ }^{25}$ Ibid., col. 277a, p. 139.

${ }^{26}$ Ibid., col. 659a-660b, p. 330.

${ }^{27}$ Ibid., col. 657 a, p. 359. 
morphologique se dessine : on peut le rapprocher de divers champs lexicaux à connotation positive, comme celui de la largesse ou de la générosité, de la prouesse, de la pitié ou de la compassion, de la noblesse, de la courtoisie, de la douceur, ainsi que de la loyauté ou de la droiture. Il est borné par les termes antinomiques mentionnés dans les oppositions vues plus haut. Ainsi, la debonnaireté s'oppose fondamentalement à la force, à toute forme de contrainte, physique ou morale, à la cruauté, à la mauvaiseté, à la trahison.

L'examen des personnages auxquels est rapportée la notion de debonnaireté est également riche d'enseignements. Elle est utilisée pour exprimer la respectabilité et pour construire la renommée des protagonistes. En dépit du caractère manichéen de la démarche, nous pouvons distinguer deux catégories de «personnages » ou de notions auxquels s'applique le concept de debonnaireté : les bien famés et les malfamés, c'est-à-dire ceux auxquels est attribuée une bonne renommée et ceux qui ont mauvaise réputation.

\section{Les bien famés}

Le paradigme morphologique de debonnaire ne s'applique pas seulement à des personnages, mais peut également caractériser des référents non humains ${ }^{28}$. Ainsi, l'on dénombre 15 occurrences de ce type. Ces référents ont des points communs : outre la personnification, ils sont présentés comme (au moins potentiellement) bénéfiques. La debonnaireté apparaît notamment comme un attribut divin, qui recouvre la bienfaisance, la générosité, la bienveillance, mais surtout la miséricorde et la clémence. Elle est aussi une des qualités essentielles du chevalier idéal, si l'on en juge par le portrait-robot que font de lui Lancelot et la Dame du Lac durant la conversation qui précède le départ du jeune homme pour la cour d'Arthur en vue d'y être adoubé. Ils y recensent les qualités morales

\footnotetext{
289 occurrences sont appliquées à Dieu ou Jésus, 3 sont attribuées à des allégories, celle de la Mort (une occurrence) et celle de la Fortune ( 2 emplois), une occurrence s'applique au pays de Gaunes, enfin deux sont employées pour un objet, l'Épée aux Étranges Attaches.
} 
(sagesse, bonté, compassion, loyauté, générosité, courage, courtoisie...) et physiques (force, beauté...) attendues d'un chevalier. Ils établissent ainsi un miroir reflétant les principes éthiques de la classe guerrière et dirigeante ${ }^{29}$. Finalement, c'est un autoportrait par anticipation que Lancelot effectue ici, puisqu'il s'avérera posséder toutes les qualités énumérées, et sera désigné de manière récurrente comme le meilleur chevalier du monde. C'est d'ailleurs lui, dans l'ensemble du princeps, qui comptabilise le plus grand nombre d'occurrences du paradigme morphologique de debonnaire, ce qui confirme quantitativement son statut de héros. La debonnaireté lui est attribuée dès l'enfance ${ }^{30}$, elle est donc présentée comme naturelle, comme constitutive de la nature même de l'enfant. Elle s'exprime dans divers domaines. Socialement, le personnage s'inscrit dans un lignage illustre et prestigieux puisqu'il est présenté comme un descendant du roi David ${ }^{31}$; la debonnaireté est donc indubitablement liée à la noblesse. Ensuite, elle se révèle par la générosité, comme lorsque, jeune garçon encore, il offre un chevreuil à un pauvre vavasseur ${ }^{32}$. Elle se manifeste également dans l'aspect physique, sa beauté ${ }^{33}$, reflet des qualités intrinsèques du personnage. Elle apparaît encore dans sa valeur chevaleresque, qu'il s'agisse du soutien qu'il apporte de manière inconditionnelle à la reine chaque fois qu'elle se trouve en difficulté, ou de la clémence dont il fait preuve à l'égard des adversaires vaincus $^{34}$, en somme lorsqu'il a le pouvoir de vie et de mort sur un autre être humain. C'est une qualité remarquable et rare, comme le souligne une demoiselle ignorant sa véritable identité : « Si en y sont passez plus de deux mille dont oncques ung seul n'en eschappa qu'il ne fust oultre ou conquis, mais il est tant

\footnotetext{
${ }^{29}$ Le procédé n'est pas sans rappeler les miroirs des princes, traités didactiques destinés à éduquer les souverains. $C f$. Einar Mar Jonsson, «Les "miroirs aux princes" sont-ils un genre littéraire ? », Médiévales, n 51, automne 2006, p. 153-166.

30 « ce fut le plus doulx enfant et le plus debonnaire la ou debonnaireté deüt estre trouvee », vol. I, col. 58b, p. 32.

${ }^{31}$ Ibid., col. 966 b, p. 490.

${ }^{32}$ Ibid., col. 60b, p. 33.

${ }^{33}$ Ibid., col. 582 b, p. 294.

${ }^{34}$ Ibid., col. 806b, p. 406 ; vol. II, col. 187a, p. 96 ; col. 484b, p. 242 ; col. 797b, p. 399.
} 
debonnaire qu'il n'en tue nul, si les eüst bien tous occis s'il eüst voulu ${ }^{35} »$. De même, son abnégation quand il renonce définitivement à son amour pour l'honneur de sa dame et la paix du royaume de Logres et de ses habitants comme de sa famille, est désignée, par deux fois, par le terme de debonnaireté : «Quant la royne oyt la conclusion et la debonnaireté de Lancelot $^{36}$ » et

Se Lancelot eüt aymé tant follement la royne comme on disoit, il ne la rendist pas si tost, car il n'est pas au dessoubz de la guerre, et pour ce qu'il a faict debonnaireté a ma voulenté, je feray ce que la royne mande du tout ${ }^{37}$.

Ces emplois illustrent la perfection morale, l'altruisme du personnage par le sacrifice de soi et de son propre bonheur au profit d'autrui.

Les proches du héros font également preuve de debonnaireté, que ce soient ses cousins Boort et Lionnel, son frère Hector, ou son grand ami le roi Gallehaut. Finalement, ce sont 32 des 105 occurrences de debonnaire et ses dérivés qui désignent Lancelot et ses proches ${ }^{38}$. Cette répartition semble en cohérence avec la hiérarchie perceptible dans l'œuvre ${ }^{39}$.

À titre de comparaison, seuls 19 emplois sont consacrés à Arthur et aux siens. Conformément à l'image traditionnelle, c'est le roi Arthur qui concentre le plus d'emplois ; 6 expriment les qualités de l'illustre souverain. Ainsi, sa

\footnotetext{
${ }^{35}$ Vol. II, col. 484b, p. 242. Questionnée par Perceval et Hector, elle dresse ici le portrait du Chevalier Meffait, pseudonyme sous lequel Lancelot se fait connaître depuis six ans, depuis que le rejet de la reine l'a mené à la folie. Sa seule occupation, sur l'île où il s'est retiré, est de jouter contre les chevaliers qui se présentent. Le discours met en relief le grand nombre de chevaliers affrontés et vaincus, mais il insiste surtout sur la grande clémence du personnage, sa magnanimité qui témoigne du grand respect que le personnage accorde à la vie d'autrui. Ce trait est particulièrement souligné par l'opposition entre nul et tous, qui montre l'exploit accompli, tant d'un point de vue chevaleresque (l'absence de défaite malgré la participation à un combat par jour, en moyenne, pendant six ans) que du point de vue humain (exploit d'être parvenu à tous les épargner).

${ }^{36}$ Ibid., col. 840a, p. 421.

${ }^{37}$ Ibid. Le locuteur est Arthur.

${ }^{38} 20$ pour Lancelot, 5 pour Boort, 4 pour Gallehault, 1 pour Ban de Benoïc, autant pour Lionnel et pour Hector.

${ }^{39}$ Par exemple, Boort, cousin germain de Lancelot, mais aussi un des trois élus du Graal, est gratifié de 5 occurrences, une de plus que Gallehault.
} 
debonnaireté est associée à sa largesse, sa sagesse, sa beauté, et sa prouesse ${ }^{40}$. C'est cette qualité morale, poussée à son paroxysme, si l'on en juge par la construction récurrente de l'adjectif précédé de l'adverbe intensif si dans la moitié des occurrences ${ }^{41}$, qui lui vaut d'être tant aimé de ses proches et de ses sujets, c'est-à-dire d'être populaire ${ }^{42}$. Les liens étymologiques entre noblesse et notoriété sont bien connus, la popularité semble en constituer une conséquence logique, liée aux actions que les grands de ce monde accomplissent, du fait de leur position et des responsabilités qui en découlent, pour ceux dont ils ont la charge. L'amour du peuple pour le couple royal est ainsi exprimé au moment où la reine est conduite au bûcher ou quand on croit le roi mort ${ }^{43}$, c'est-à-dire dans un contexte de lamentation. Guenièvre et Gauvain sont à leur tour caractérisés par la debonnairetét ${ }^{44}$. La reine est présentée de manière élogieuse ; le texte souligne sa capacité à pardonner la trahison des barons durant l'épisode de la fausse Guenièvre, mais aussi son soutien aux nécessiteux par le recours à l'hyperbole ${ }^{45}$. Gauvain, le neveu préféré d'Arthur, se trouve en troisième position dans la fréquence des emplois du paradigme morphologique, puisque lui sont attachées trois occurrences. Cependant, à y regarder de plus près, il apparaît que toutes sont

\footnotetext{
${ }^{40}$ Vol. I, col. 51a, p. 29 et col. 52b, p. 29 ; vol. II, col. 336b, p. 168 et col. 851b, p. 426.

${ }^{41}$ «Si debonnaire » : vol. I, col. 51a, p. 29 ; vol. II, col. 336b, p. 168 et col. 851b, p. 426.

${ }^{42}$ D'autant que ce jugement positif sur le roi est à plusieurs reprises attribué à d'autres personnages par le biais de focalisation zéro ou de paroles rapportées, y compris provenant de protagonistes ennemis d'Arthur.

43 « Haa ! Douce dame debonnaire, ou trouveront jamais povre gent pitié ne debonnaireté ?» (vol. II, col. 811b) ; «Et quant la nouvelle fut respandue par la cité et on creüt que le roy estoit occis, qui tant estoit preudhomme et qui leur avoi testé si doulz et si debonnaire, ilz en firent moult grant dueil » (vol. II, col. 851b).

${ }^{44}$ Guenièvre est gratifiée de 5 occurrences, Gauvain de $3 ; 2$ occurrences se rapportent à Yvain, autant au duc de Clarence, et une à Saigremor le Desréé.

${ }^{45}$ Dans la quasi-totalité des cas, les occurrences sont accompagnées de l'adverbe intensif moult ou d'une formulation hyperbolisante, que ce soit une tournure exceptive («en tout le monde tant comme il dure, ne a encores aussi vaillant dame ne aussi debonnaire aux povres chevaliers comme elle est », vol. II, col. 795b, p. 398) ou le recours à la tournure interrogative associée à l'adverbe jamais («Haa! Douce dame debonnaire, ou trouveront jamais povre gens pitié ne debonnaireté ?», vol. II, col. $811 \mathrm{~b}$, p. 406) qui souligne sa singularité, ses qualités exceptionnelles.
} 
constituées de l'adverbe debonnairement ${ }^{46}$ par conséquent, la debonnaireté caractérise une manière d'être, un comportement, non une qualité intrinsèque du personnage. Ce constat entraîne une révision de la psychologie du personnage, bien moins lisse et bien moins positif qu'on aurait trop rapidement tendance à le croire, souvent considéré comme un modèle de courtoisie, mais dont l'expertise dans le jeu social et le comportement de séducteur assez mécanique dévoilent aussi la part d'ombre. Tout cela rend plus cohérente sa déchéance finale dans la haine de Lancelot, l'aveuglement, le désir insatiable de vengeance, en somme dans l'hybris, que les médiévaux appellent outrage, et qui contribue largement à la destruction du royaume de Logres.

\section{Les mal famés}

Le roman met cependant en scène des personnages mal famés. Il s'agit des opposants, des personnages malfaisants, principalement, par ordre d'importance (dans le récit comme dans le nombre d'occurrences), de Claudas, Mordret, Méléagant et de la fausse Guenièvre ${ }^{47}$. On ne rencontre aucune occurrence de l'antonyme «habituel» de debonnaire, à savoir l'adjectif deputaire ou l'expression plus archaïque et non agglomérée de pute aire. Ce sont donc l'adjectif debonnaire et le substantif debonnaireté qui sont utilisés pour dénier cette qualité à ces personnages. Ainsi la messagère de la Dame du Lac, quand elle s'adresse au roi Claudas auquel elle vient reprendre Boort et Lionnel, lui conteste la détention de cette qualité : «l'en lui avoit dit que vous estiez le plus gentil roy et le plus debonnaire du monde, le plus vertueux, le plus large, et de la plus haulte prouesse $^{48} \gg$. Elle emploie également la négation pour exprimer cette contestation :

\footnotetext{
${ }^{46}$ Vol. I, col. 326b, p. 166 ; col. 369a, p. 188 ; col. 933a, p. 470.

${ }^{47} 8$ occurrences concernent Claudas, 2 désignent Mordred, autant Méléagant, et une la fausse Guenièvre.

${ }^{48}$ Vol. I, col. 75a, p. 41. En témoignent l'utilisation du passé et la prise en charge de ce jugement positif par l'indéfini l'en.
} 
« vous avés failly a troys des meilleures vertus qui puissent estre en chevalier, car vous n'avez ne sens, ne debonnaireté, ne courtoisie ${ }^{49} »$.

La caractérisation des autres personnages mal famés fonctionne sensiblement de la même façon, par la négation ou la remise en question de la debonnaireté du personnage, par exemple par l'utilisation du futur 2, à valeur hypothétique ${ }^{50}$. Mais pourquoi recourir à la périphrase plutôt qu'au terme deputaire? Sans doute parce que le sème étymologique de noblesse demeure, malgré le développement d'autres sens, un sème essentiel de debonnaire, tandis qu'il est totalement rejeté par l'antonyme. Or, ces personnages appartiennent à la haute aristocratie puisqu'ils sont rois ou fils de rois. Le princeps semble rechigner à franchir le cap de l'irrévérence ${ }^{51}$.

La notion de debonnaireté apparaît dans les situations de négociation, et dans les menaces, elle y constitue l'argument avancé comme l'ultime étape avant l'escalade de la violence ${ }^{52}$. Mais la notion est galvaudée dans les discours de personnages mal intentionnés. C'est le cas par exemple dans les dialogues qui précèdent un combat ${ }^{53}$. Elle sert ainsi à justifier la contrainte exercée sur autrui ${ }^{54}$. Enfin, dans des paroles d'intimidation, elle constitue l'un des deux éléments d'une

\footnotetext{
${ }^{49} \mathrm{Ibid}$.

50 «par ce avriez les cueurs de maintes gens et vous tiendroit l'en pour saige, courtois et debonnaire », ibid. ; «Filz, dit le pere, tu y avras plus de honte que de blasme, car les gens diroient que tu lui avroies rendue par ta debonnaireté ce que tu avoies conquis par ta prouesse », ibid., col. 769a, p. 388.

${ }^{51}$ D'autant que, au moins en ce qui concerne Claudas et la fausse Guenièvre, les caractères présentent des nuances. Ainsi, Claudas est capable de reconnaître la grande valeur d'Arthur, il éprouve même respect et tendresse envers certains de ses hommes, en particulier ceux qui osent lui tenir tête et faire preuve d'intégrité, comme le fait notamment Farien. La fausse Guenièvre, quant à elle, se repent de ses méfaits à l'article de la mort.

${ }^{52}$ C'est ce qui se produit lorsque Farien, tuteur de Boort et Lionnel, exige du roi Claudas, qu'il croit responsable de leur disparition, qu'il les lui rende, l'exhortant à adopter une conduite conforme à son statut royal : «se debonnairement ne les nous rendez, il vous convendra tous mourir, vous et vostre compaignie » (ibid., col. 88b, p. 63).

${ }^{53}$ Comme dans les propos qu'un chevalier adresse à Gauvain avant de l'attaquer : «puis que vous ne le voullez faire par debonnaireté, vous le ferez par force » (ibid., col. 942b, p. 474).

${ }^{54}$ Ainsi est avancée l'explication que la fausse Guenièvre donne de son comportement : «et pour ce que vous estes le plus preudomme du monde vous ay-je prins par force quant je ne vous povoie avoir par debonnaireté » (ibid., col. 570b, p. 288).
} 
alternative abjecte, l'autre étant la violence physique. Ce détournement de valeur se fait de surcroît au détriment de ceux que l'aristocratie a normalement pour mission de protéger, notamment les femmes ${ }^{55}$. Les chevaliers dévoyés procèdent donc à un renversement des valeurs de leur classe pour justifier leurs agissements, comme pour maintenir l'illusion de leur respectabilité. Les manquements les plus graves à l'éthique apparaissent dans le second volume, lorsque la chevalerie est sur le déclin.

Pour conclure, la debonnaireté malgré son importante polysémie, apparaît comme une notion fondamentale dans l'editio princeps, où elle est attachée à des valeurs sociales et morales fortes, qui renvoient à la noblesse et à la bonté, c'està-dire aux notions de réputation et de respectabilité qui construisent la renommée et justifient l'importance accordée au personnage de Lancelot dans cette version tardive. La pérennité de sa présence montre que le héros conserve encore, en cette fin de $\mathrm{XV}^{\mathrm{e}}$ siècle, ses caractéristiques médiévales et son statut de modèle, ce qui contribue à expliquer les huit éditions qui ont été produites entre 1488 et 1591 , mais aussi peut-être la désaffection dont il fait ensuite l'objet, par obsolescence des valeurs dont il est le parangon.

\footnotetext{
${ }^{55}$ C'est le cas lorsque Claudas et ses sbires entreprennent indûment de mettre la main sur des lettres qu'une messagère de la reine Guenièvre a envoyées à Lancelot (« Damoiselle, vous estes venue en message en ce pays, et pour ce que nous ne sçavons se vous estes venue pour nostre bien ou pour nostre mal voullons-nous veoir les lettres que vous portez, si vous prie tant comme je puis que les baillez debonnairement avant que l'en vous face force », vol. II, col. 237a, p. 119 ; «Et se debonnairement ne les voulez mostrez, je feray tant cercher vous et vostre compaignie que l'en les trouvera, si les verrons mal gré vous », ibid.) ou, pire encore, dans les pressions psychologiques exercées en vue d'aboutir à un viol (« Damoiselle, fist-il, vous direz tout ce qu'il vous plaira, et je vous escouteray, mais toutesvoyes vous prie-je que vous faciez ma voulenté debonnairement, et ne soyez pas si villaine qu'il vous convienne faire force, car aprés ne vous aymeroie pas, et aussi vous voyez bien qu'il est force que vous le faciez, car la force n'est pas vostre, et mon vouloir y est », vol. II, col. 14b, p. 9 ; « il print la damoiselle et la jetta soubz lui en lui disant qu'il luy feroyt force se elle ne le vouloit ottroïer debonayrement », ibid.).
} 
